\title{
"No Lecture, I Am Bored:” What Messages does Young Males Myanmar Want to Learn from Sexuality Education in Social Media?
}

\author{
Nan Yu Khine* Pimpawun Boonmongkon Penchan Pradubmook-Sherer \\ Faculty of Social Sciences and Humanities, Mahidol University, Thailand \\ *E-mail of the author: nannyu.mdy@gmail.com
}

\begin{abstract}
This paper explores how Myanmar male youths use the social networking sites especially Facebook to search sexual health information by investigating their pattern of usage and the types of sexual message they are eager to learn from online source. The study was undertaken by conducting virtual ethnography, 16 in-depth interviews and 3 FGD sessions through purposive sampling method among university students in Mandalay. Thematic analysis was guided by doing open coding. Among male youth community, searching sexual health information and seeking sexual pleasure from Facebook are very common and are main reasons to use Facebook. In conclusion, Facebook influences their sexual health in both positive ways and negative ways depending on their pattern of usage. It is recommended that young people need sexual health information and comprehensive sexuality education curriculum in schools that are related with their sexual interest, sexual culture and lifestyle and their sexual literacy.
\end{abstract}

Keywords: Pattern of Facebook Usage, Sexual Health, Male Youths, Myanmar

DOI: $10.7176 /$ RHSS/11-14-02

Publication date:July $31^{\text {st }} 2021$

\section{Introduction}

Nowadays, in our daily life, all of interactions with our family, friends and neighborhood and everything in our environment had been changed by the internet. Someway, for both our personal and professional lives developments, we used internet as an essential technology applications in our modern era. We achieved by using the internet in various different ways imperceptibly. In addition, the internet was an essential thing to keep in touch with family members, relatives, colleagues and friends who are living apart from us and to meet new people all around the world (Lister, Giddings, Dovey, Grant, \& Kelly, 2008).

Globalization bringed advanced technology and internet access to Myanmar. Because of that Internet has been accessible since 2000. But, using internet is very high charges and restircts rules and regualtions, cyber law, infrastructure and software based censorship. Regardless of these barriers, Internet access promoted social networking to be a popular medium in communication among urban citizens mostly in Yangon and Mandalay(Lin \& Van der Putten, 2012). In Myanmar, there are 16 million people who are using the internet. Among that, 14 million people (87\%) are youth between 13 to 34 years of age. According to social media usage analysis, 96.29\% of Myanmar people use Facebook, 1.43\% use Pinterest, 1.1\% use Twitter and etcetera. According to that data, Myanmar people use Facebook as main social media(Myanmar, 2017).

It is obivous this technological revolution also transmitted to Myanmar Youth society and a lot of users generated sexual or pornographic content sharing groups and web pages were developed among youth. These web pages and groups are popular and growing with a lot of members and attracted new members. The most popular web pages and groups for sexuality are Adult's Way Out (X squad) with 20k members, Gong Sing Groups with 19k members, Ah Hmaung LawKa(Dark Life) with 20k members as well as others. The word "Gong Sing"(Freeloaders) $)^{l}$ is most popular slang being used in the social media, especially in the facebook. Facebook is the most population application for most young Myanmar men and they can talk, discuss, learn and share about sex with free of charges by joining their own social groups or networks in facebook Facebook is a mean of application that everyone use in their every day lives. At Facebook, there are over 50 Gong Sing sex groups and have average around 5k members. Among Youths, many pages and groups to talk and discuss about sex are developing day by day and people are enjoying to join these groups.

In Myanmar, even though sexuality education curriculum in primary, middle and high schools are appointed by government programs, the implementations of these programs are still weak. In addition to that, parents, community, and schoolteachers are reluctant to discuss sexual topics openly and provided little or suspect information. In the society, taking and discussing about sexuality among adolescent and young people is very sensitive. In the community, it is a cultural taboo that prevents the dissemination of any information about sex. There are regular school- based SRH education classes in some parts of urban areas, so youths can get SRH

\footnotetext{
${ }^{1}$ Gong Sing.........User Generated Sexual or Pornographic Content Sharing Web Page/Group at Facebook.
} 
information from these classes, but out-of-school youths cannot access this information. Adolescents do not want to discuss easily sensitive issues with someone they meet on daily basis. So, youths have an obviously lower accessibility and unmet needs for SRH information (UNFPA, 2005).

Studies have shown that the word "sex" is the most popular "search term" used on the Internet social media today (Pastore, 2001). Youths have turned to the Internet and social media in search of knowledge and to discuss their sexual experiences, they get the require additional information or emotions about sex that they are looking for, intimacy sexual feeling and relationships (Ngo et al., 2008). The youths use the social media like Facebook for their identity formation, identity development by participation on media based youth subculture and participating in their socialization because they have significant freedom and choice at media (Arnett, 1995).

Young men and women cited the internet to inquire and improve their existing knowledge, especially sexual literacy obtained from their conventional sources such as family, parents, and printed media. Their society gives the little information related with sexual education (Ngo, Ross, \& Ratliff, 2008). In addition, the Internet can have positively impact on youths' sexuality easily and allows for the broadcasting of information about worldwide(Cooper, 1998).

The Internet might have both positive and negative effects on sexual health. According to one national survey of young people (10-17 years old) who regularly used the Internet, one out of four said he or she had encountered unwanted pornography in the past year, and one out of five had been exposed to unwanted sexual solicitations or approaches (Finkelhor, Mitchell, \& Wolak, 2000). At the same time, a number of sites, such as the American Social Health Association's iwannaknow.org, promote healthy sexual behavior and provide young people with advice on communication in relationships as well as methods for protecting against sexually transmitted diseases (Jane D. Brown,2002).

To date no study has been done about influences of social media (Facebook) usage on sexual health among young male in Myanmar. The study aims to study the pattern of Facebook usages and what types of messages they learn from online Facebook source. Within the contexts of worrisome of sexual health needs among young males in Myanmar, understanding the pattern of social media (Facebook) usage related with sexual health among young male in Myanmar will be useful for those committed to advocate for sexual health and wellbeing of this socially neglected subculture to explore avenues to reach the youth community in Myanmar.

\section{Methodology}

In this study, the author used qualitative and virtual ethnographic approach, in which the researcher involves in constructing and narrating such reality by sharing the experiences with youths in online space. This study was undertaken by conducting in-depth interviews with male youths and online participant observation in three selected users generated sexual and pornography content groups at Facebook. Among male youths attending universities and youth centers at Mandalay 16 participants were be purposively selected, based on inclusion criteria (male, Myanmar national, 18 to 24 year of age, Facebook active user, member of users' generated sexual and pornography content groups at Facebook) and exclusion criteria (age below 18 years and above 24 years, Female, inactive Facebook user, non-member at users' generated sexual and pornographic contents group of Facebook), through assistance of authorities from universities and youth centers by a snowball sampling technique.

In this study, virtual ethnography with online participant observation was conducted. Online participant observation was done by participating selected online groups and observation their online activities (discussions, posts, content preferences, activities) at nighttime between $8-10 \mathrm{pm}$ during 2 month of data collection period. Individual in-depth interviews were conducted through Facebook messenger by using semi-structured questionnaires because of informants' preferences. The young males in this study preferred to conduct in-depth interview through online Facebook messenger texting than face to face in-depth interview due to gender and sexuality issues being involved. It is difficult to talk about sexuality topic between those who are difference gender. The cultural barrier created embarrassment between difference gender of researcher and informants during interviewing more intensively about sexuality topic.

Ethical clearance was obtained from Mahidol University's Human Research Ethics Committee (approval project No. 2018/171B). Informed consent of all participants was obtained. Personal identifiers were removed from interview reports to ensure confidentiality, whereas a passport protected electronic logbook was maintained. Interview guides were translated from English into Myanmar language and reports translated back into English language by independent translators.

\section{Results}

\subsection{Socio Demographic Characteristics and Pattern of Facebook Use}

Study informants consisted of 16 young male with the age range 18 to 24 . Among them, 3 young males are already graduated and the other 13 are university students. Some of the graduated young males are working in marketing industry, telecommunication industry and some are attending the trainings. They all lived in Mandalay, 
the second largest city of Myanmar for their job and study. All of participants are single and lived in hostels near their university. They used Facebook since they finished their high school and are active Facebook users who participate in users generated sexual and pornographic contents groups at Facebook.

\begin{tabular}{|l|l|}
\hline Variable & Percentage \\
\hline Age & $13(81 \%)$ \\
$18-20$ years & $3(19 \%)$ \\
\hline $21-24$ years & $16(100 \%)$ \\
\hline $\begin{array}{l}\text { Gender } \\
\text { Male }\end{array}$ & $16(100 \%)$ \\
\hline Sexual Identity & $13(81 \%)$ \\
Heterosexual & $3(19 \%)$ \\
\hline Education & \\
Students & $16(100 \%)$ \\
\hline Graduate & $2(13 \%)$ \\
\hline Marital Status & $14(87 \%)$ \\
\hline Single & \\
\hline Employment status & $2(100 \%)$ \\
Working & \\
Not Working & $16(100 \%)$ \\
\hline Occupation & \\
\hline Private Employee & \\
\hline Living Status & \\
Hostel & \\
\hline
\end{tabular}

a. Pattern of Facebook use related to sexual health among male youth community

In this study, pattern of Facebook usage means frequency of their usage, their amount of spending time at Facebook, what age do they start using it, time of using it in their life, the purpose of FB usage and the utilities of FB in their life including social networking, finding friend, etc.

The young people from in this study, most of them started using FB after 2010 because Myanmar government had made restriction of telecommunication market that led to high charges of mobile sim card before the year 2010. In 2010, the new government reduced restrictions on charges of mobile sim card, rules, and regulations of cyber usage by opening the telecommunication market. So, most of the people can easily afford to use mobile phone and access internet. Since then, FB becomes the most popular social media in Myanmar.

In this study, majority of young people had started to use mobile phone at their parents' houses and during university attendance after they finished their high school education. After finishing the high school education, their parents gave a mobile smart phone as an award. Internet can access anywhere and anytime. Handsets and data package for internet use are affordable for them.

Almost all of youths use FB with many purposes and participate in many online activities. So, FB becomes very popular application and source for knowledge and entertainment for them. Most of young people have 2 to 4 Facebook accounts and use FB average $2 \mathrm{hr}-4 \mathrm{hr}$ per day. The peak time of Facebook using is between $8 \mathrm{pm}$ to $12 \mathrm{pm}$ at night.

The study indicated that the meaning of FB in their lives is for their knowledge and entertainment. For knowledge, they wanted to get update news locally and globally, general knowledge especially sexual health knowledge. For entertainment, they used for social interaction such as contacting with old friends, making new friends, listening to music, getting sexual pleasures from sexual contents and pornographic images, videos at FB. So, it can be explained into three meanings depending on their purpose of usage as seeking knowledge about sexual health and sexuality, social interaction and seeking sexual pleasure from sexual contents at Facebook.

\subsubsection{Seeking knowledge about sexual health and sexuality}

All of informants use FB as their main social media and they all agreed FB search is their most common access point for sexual health information. In this study, only 2 informants sometimes use Google search related to sexual health information.

The informants said that

"FB is a main source of knowledge for me" (Thant)

"I am very good enough in using FB more than Google searching because I am very familiar with Facebook and 
all of my friends use Facebook" ( kyaw kyaw)

They use FB related to sexual health because FB is very familiar for them and can use FB without any technical barrier as well as all their friends also use FB.

One participant said that

"nowadays we learn about sex from FB, before FB we learned about sex from watching porn movies, reading porn literature, asking from friends and brothers. ' ( Kaung Kaung)

\subsubsection{Types of sexual Message}

Moreover there are many sexual contents in FB and it is very easy to search any information about sex. There are too many user generated sexual content groups and pages that everyone can join and participate without any cost. If they posted any questions or discussions about sex in these groups, too many members give answers and suggestions.

The informant mentioned that

"You can search any information by typing keywords at search bar, how easy" (Shin Shin)

"I like to learn from discussions of these groups; I can get this opportunity at only FB" (Kyaw Kyaw)

Furthermore, the young people have desire to get entertainment in learning about sex. At FB, most of sexual contents such as discussions, porn literature and porn movies give entertainment to them. Actually, they know the official pages for sexual health information from NGOs or GOs people, but they don't join because they don't want to get serious messages. They think that the messages from official or professional social network was over whelmed with lots of information that lack excitement but boring and were not relevant to their interest and their sexual lifestyles Hence, they prefer to learn about sex from user generated sexual content groups and pages.

The informant said that

"I think no one want any lecture while using FB" (Thant)

"The official pages give information too much, I am boring to read" (Kaung Kaung)

They searched information about sex through FB search bar and users generated sexual contents groups and pages. They know to join these groups by sharing from their friends and popular across at their newsfeed; sometimes they added as member each other in respective groups. They can choose these groups to join with their content preferences. There are many different types of users' generated sexual contents groups and pages with different contents such as sexual pleasure, health problems related to sex. All of them joined average 5 to 10 users' generated sexual content groups and pages for both sexual pleasure and sexual health problems.

They learned about sexual knowledge from different sources of sexual contents such as discussion, sex experiences of members, watching porn movies and reading porn literature. In these groups, they can learn with their own preferences and interests about sex. The young people want to know how to start sexual conversation with their girl friend, how to have the ability to attracts sexually to their partners and the nature of female sexual desire and sexual pleasure. Most of the problems and discussions in these groups are pregnancy case after sex, sign and symptoms of sexually transmitted diseases and how to enlarge their penis size and be sexually attractive to their sexual partners.

In this study, they discussed what their preferences and how they learned about sex from different sexual contents at FB,

"I want to know the emotions, feelings of female during their menstrual period, If I know, it will be easy to communicate with my girlfriend" (Zaw Zaw)

"For me, I learn the methods of condom usage and contraceptive drugs" (Khat Lwin)

"Yah, FB is main source to get information about sex workers, we can easily contact them" (Lin Lin)

\subsubsection{Pattern of Facebook use for seeking sexual pleasure}

Seeking sexual pleasure is one of their sexual purposes in using Facebook. Most of the users' generated sexual groups are created to share sexual contents for sexual pleasures. They shared pornographic literature, sexy photos and porn videos from Asians and around the world and doing sex chatting with girls. The Japanese porn videos are so famous among young community. Their preferences for sexual contents are homemade sex videos of couple from Myanmar. The word "Lin Pay Par" that means "share with me" is common word in requesting sexual contents in these groups.

They find the partners for sex chatting at Facebook. There are many girls who provide sex chatting services with money. The young males have to pay moneyaround 5000-10000 MMK (5\$-10\$) to girls for sex chatting. Sometimes, they got the partners from their friend list and don't need to pay money. The informant said that

"I did sex chatting with the girls in my Facebook friend list, actually we are just friends, I don't need to pay money” (Htun Win)

"In these groups, we shared the contact information of girls who provide sex chatting services with money" (Aung)

They have many Facebook accounts and separated accounts in using. They used Facebook with real profile and fake profile alternately. When they have sexual desire, they usually open fake account for sexual pleasure. They 
used Facebook with fake account /fake profile for sexual purpose because of their subjectivities on constructed cultural barriers.

\subsection{Pattern of Facebook use for social interaction}

The young people used for social interaction such as contacting with old friends, making new friends, speaking with relatives. They search their old friends from their high school and put them each other to the Facebook friend list. In this way, they keep in contact through Facebook. And they make new friends with strangers. Among them, making new friend with beautiful and sexy girls feel them proud to be manly.

They got girlfriends with strange girls by flirting at Facebook. Facebook reactions and features make users to close each others. They take care each other by giving reactions such as "like", "Love", "Wow", "Sad" and "Angry" and comments to posts and photos at Facebook. In online culture, it is very popular that young males write the romantic comments by competing each other below beautiful and sexy girls' posts or photos. It makes entertain them by showing how much they are romantic. Most of the comments that they give are

"I wish you were my girlfriend"

"My girlfriend be like"

"Trust me; I will be your romantic boyfriend"

"I give you my whole life"

"Can we take through private chat box?"

The informants said that

"I met with my girlfriend from Facebook" (Kyaw Kyaw)

"I have a lot of Facebook friends, this make me happy"

"When I post something, I got many reactions and comments from my friends, I really enjoy" (Thit)

They have many groups with different topics at Facebook. They created many groups with many purposes eg. studying, to share music songs, charity activities, sexual related groups. They join and participate in these groups for sharing information, discussing opinions and social networking. The popular Facebook groups are "Book lovers", "Music addicted groups", "The people who like music songs", "People who search general knowledge", etc. The users who have same interests, they created groups and discuss the issues.

\section{Discussion}

Almost all of informants use the Facebook to improve their sexual literacy and sexual pleasure. It is obvious that they improved their knowledge related to sexual health by using Facebook. They gained knowledge on condom usage, how to use condom and advantages of condom usage, the importance of consent in sexual communication from the users' generated sexual and pornographic content groups that they joined. At the same time, there are several gender norms and sexual norms shared by members in these groups. These gender norms lead to male dominant power relationships in sexual communication and sexual norms also lead to misconception of protective sex and responsibility sex. This need to be addressed even though their sexual health knowledge increased, their sexual practices were influenced by the gender norms and sexual norms from these groups.

Among young male community in Myanmar, talking and discussing about sex is still silence. The informants from this study refused researcher to do face to face interview and requested to do interview through FB messenger. Being female researcher, they are embarrassing to talk about sex because of gender and they feel comfortable to do through messenger. Communication through messenger is considered to be their online culture.

Young males are not interested in lecture based sex education given from NGOs and government officials at Facebook. In their thinking these information are too serious and lack of excitement. They like to search information with their curiosity by asking questions themselves. They want to learn sex education with discussion styles by sharing their sexual experiences.

As discussed in earlier work (Ngo et al., 2008), internet become peer education forum regarding about sex for youths and acts a main source to get the knowledge regarding sex by talking, discussing, sharing about sex for youths(Arnett, 1995), our findings also demonstrated all of informants ranked FB is the first point that they searched sexual health information and sexual pleasure in their online culture.

Young people overcome cultural barriers by using the online communication for discussion of these issues, leading to the sharing of these inner side feelings that increases their intimate performances (Ngo, Ross, \& Ratliff, 2008). In our study, the informants revealed that Facebook make them to reduce their worries related to cultural taboos and encourage to share their sexual experiences and discuss about sex.

The argument for this study was on the work of (Anh D. Ngo,2008), the youths change their sexual behaviors from traditional norms to modern norms or international norms by learning the sexual behaviors of western youths and other media contents (movies, music) by using social media. Our finding demonstrated that in the users' generated sexual and pornographic content groups at Facebook, the gender norms and sexual norms are more constructed by sharing their sexual experiences and perceptions among members. 


\section{Recommendations}

It could be seen young people are struggled to get sexual health information and knowledge in their life since Myanmar society are reluctant to discuss about sex. There is needed sex education curriculum in school to provide sexual health knowledge. Sexual and reproductive health services are inaccessible for young people. The researcher recommended that it is needed to educated gender norms and sexual norms to young people. In sex education curriculum, knowledge only about sex is not enough and should include about gender equality, gender norms and sexual norms. At Facebook, fake profiles, privacy breaks, financial fraud and cyber bulling are very common among young people. The government should implement the policies to decrease these challenges. Future research should be conducted why young people prefer users generated sexual groups than the sexual and reproductive education pages created by NGOs and government officials.

\section{References}

Arnett, J. J. (1995). Adolescents' uses of media for self-socialization. Journal of Youth and Adolescence, 24(5), 519-533.

Brown, J. D. (2002). Mass media influences on sexuality. Journal of sex research, 39(1), 42-45.

Cooper, A. (1998). Sexuality and the Internet: Surfing into the new millennium. CyberPsychology \& Behavior, 1(2), 187-193.

Finkelhor, D., Mitchell, K.J., \& Wolak, J. (2000). Online victimization: A report on the nation's youth. Alexandria, VA: National Center for Missing and Exploited Children. (CV38)

Lin, K. S., \& Van der Putten, M. (2012). Identities in motion: cyberspace and Myanmar men having sex with men. Research on Humanities and Social Sciences, 2(4), 36-48.

Lister, M., Giddings, S., Dovey, J., Grant, I., \& Kelly, K. (2008). New media: A critical introduction: Routledge. Myanmar, S. S. M. (2017). Demographic Surveys.

Ngo, A. D., Ross, M. W., \& Ratliff, E. A. (2008). Internet influences on sexual practices among young people in Hanoi, Vietnam. Culture, Health \& Sexuality, 10(S1), S201-S213.

Pastore, M. (2001). Search engines, browsers still confusing Many Web users. CyberAtlas.

UNFPA. (2005b). Programme assessment and review (2002-2005). 